\title{
ALTERED CYTOKINE PRODUCTION BY SPECIFIC HUMAN PERIPHERAL BLOOD CELL SUBSETS IMMEDIATELY FOLLOWING SPACEFLIGHT
}

\author{
Brian E. Crucian', Michael L. Cubbage ${ }^{2}$ and Clarence F. Sams ${ }^{3}$
}

${ }^{1}$ Wyle Laboratories, Cell and Molecular Research Laboratories, Houston, Texas; ${ }^{2}$ Baylor College of Medicine, Pediatrics-Hematology Oncology Department, Houston, Texas; ${ }^{3}$ NASA-Johnson Space Center, Cell and Molecular Research Laboratories, Houston, Texas

Address reprint requests to: Clarence F. Sams, Ph.D., NASA-Johnson Space Center, Life Science Research Laboratories, Mail Code SD3, Houston, Texas 77058, ph: 218-483-7160; fax 281-483-2888 email: csams@ems.jsc.nasa.gov 


\section{ABSTRACT}

In this study, we have attempted to combine standard immunological assays with the cellular resolving power of the flow cytometer to positively identify the specific cell types involved in spaceflight-induced immune alterations. We have obtained whole blood samples from 27 astronauts collected at three timepoints $(\mathrm{L}-10, \mathrm{R}+0$ and $\mathrm{R}+3)$ surrounding four recent space shuttle missions. The duration of these missions ranged from 10 to 18 days. Assays performed included serum/urine cortisol, comprehensive subset phenotyping, assessment of cellular activation markers and intracellular cytokine production following mitogenic stimulation. Absolute levels of peripheral granulocytes were significantly elevated following spaceflight, but the levels of circulating lymphocytes and monocytes were unchanged. Lymphocyte subset analysis demonstrated trends towards a decreased percentage of $T$ cells and an increased percentage of $B$ cells. Nearly all of the astronauts exhibited an increased CD4:CD8 ratio, which was dramatic in some individuals. Assessment of memory (CD45RA+) vs. naïve (CD45RO+) CD4+ T cell subsets was more ambiguous, with subjects tending to group more as a flight crew. All subjects from one mission demonstrated an increased CD45RA:CD45RO ratio, while all subjects from another mission demonstrated a decreased ratio. While no significant trend was seen in the monocyte population as defined by scatter, a decreased percentage of the CD14+CD16+ monocyte subset was seen following spaceflight in all subjects tested. In general, most of the cellular changes described above which were assessed at $R+0$ and compared to $L-10$ trended to pre-flight levels by $R+3$. Although no significant differences were seen in the expression of the cellular activation markers CD69 and CD25 following exposure to microgravity, significant alterations were seen in cytokine production in response to mitogenic activation for specific subsets. T cell (CD3+) production of IL-2 was significantly decreased after at $\mathrm{R}+0$ as was IL-2 production by both CD4+ and CD8+ T cell subsets for most subjects. Production of IFN $\gamma$ did not appear to be affected by microgravity exposure in either $\mathrm{T}$ cells in general or in the CD8+ T cell subset. There was a spaceflight-induced decrease in IFN $\gamma$ production in the CD4+ $\mathrm{T}$ cell subset, however it did not reach statistical significance. Serum and urine stress-hormone analysis indicated significant physiologic stresses in astronauts following spaceflight. In summary, these results demonstrate alterations in the peripheral immune system of astronauts immediately after spaceflight of 10 to 18 days duration and support continued research regarding microgravity and immunology (including in-flight sampling) prior to routine long-term spaceflight for astronauts. 


\section{INTRODUCTION}

Dysregulation of the immune system has been a well-documented effect of human exposure to a microgravity environment during spaceflight (1). Extensive ground based studies using clinorotation cell culture, animal restraint unloading and human long term bed-rest have demonstrated a variety of the effects of simulated microgravity on the immune system. These effects have included altered cytokine production $(2,3,4)$, reduced proliferative/activation responses $(5,6)$ and altered signal transduction pathways $(5,7)$. In addition to these ground based models, studies performed on subjects exposed to the space environment during and immediately after landing have also demonstrated altered immune responses (8). These data have included altered cytokine production $(9,10,11)$ and altered distribution of peripheral immune cells $(12,13)$. Recent reports have documented immunological studies performed in-flight (14). When tested during spaceflight, delayed type hypersensitivity was reduced, indicating a dysregulation of cellmediated immune function $(\mathbf{1 5 , 1 6 )}$. The mechanisms which produce this dysregulation have not been clearly established. In addition to the direct effects of microgravity on cellular physiology other factors may also have influence. These may include radiation, changes in calcium usage in the musculosystem and psychological issues such as stress.

The production of cytokines plays a critical role in the ability of a host to mount an immune response against an invading microorganism. In addition, the particular cells producing cytokines and the specific cytokines produces directly determine the type and magnitude and duration of the immune response. It naturally follows that a decreased ability to produce cytokines can result in potentially significant immune alteration and a reduced ability to fight infections.

In the 1990s the ability to detect cytokine production in individual positively identified cells by flow cytometry was developed $(\mathbf{1 7 , 1 8 , 1 9 )}$. Conventional assays to detect cytokine production have several drawbacks. Bioassays are not necessarily cytokine-specific in that they measure functional properties. Supernatant cytokine protein can be readily measured by ELISA methods, but unless a highly purified cell population was cultured this method does not identify the population of cells responsible for the cytokine production (i.e.: PBMC's). In addition, the results of ELISA assays reflect the net outcome of produced, absorbed and degraded cytokine and do not necessarily distinguish between biologically active and inactive substances. In situ hybridization and RT-PCR detect cytokine gene expression and are highly sensitive but they are labor-intensive and the presence of cytokine RNA does not guarantee translation to cytokine protein. The advantages of this flow cytometric detection of cytokines are the detection of cytokine protein, as opposed to cytokine RNA and the ability to examine the production of cytokines by individual cell populations while still culturing mixed populations of cells (PBMCs) to retain the various cell-cell interactions. The assessment of cytokine production has been shown to have great value in the research laboratory for investigating basic biological interactions $(20,21,22)$. Recently, flow cytometric cytokine detection has been demonstrated to have diagnostic value in clinical situations $(23,24)$ and will conceivably enter into clinical use for the rapid and routine monitoring of cytokine production and peripheral immune balance.

In this study, we have attempted to combine standard immunological assays with the cellular resolving power of the flow cytometer to positively identify the specific cell types involved in microgravity induced immune dysregulation. Blood was collected from 27 astronauts (representing four recent space shuttle missions) both before launch and at 2 points after landing. A comprehensive battery of standard immunological assays was performed, in addition to the detection of cytokine responses by flow cytometry. Due to the limitations in available sampling 
for each flight all assays were not performed on all shuttle flights, however all assays were performed on samples from at least two of the four shuttle flights (intracellular cytokine assessment was performed on three flights). We found that the ability to produce IL-2 was depressed after spaceflight, and that CD4+ and CD8 + T cell subsets seemed equally sensitive to the effect. Regarding IFN $\gamma$ production, only the CD4+ T cell subset demonstrated decreased production after spaceflight. IFN $\gamma$ production by the CD $8+$ subset appeared largely unchanged.

\section{MATERIALS AND METHODS}

Whole blood collection. Whole blood was collected from 27 healthy astronaut donors at three timepoints surrounding space shuttle flights. These subjects consist of the entire crew from four separate shuttle missions, which varied in length from 10 to 18 days. Blood collection was obtained 10 days prior to launch (L-10), immediately after landing $(R+0)$ and three days after landing $(L+3)$. The whole blood was collected into EDTA Vacutainer tubes (Becton Dickinson, Mountain View, CA). Informed consent was obtained from all participants.

Hematology analysis. Standard clinical hematology analysis was performed according to the manufacturers instructions on a Coulter MaxM hematology analyzer. Results obtained included a standard complete blood count and WBC differential, including cell percentages and absolute counts.

Cell culture for activation. Four $\mathrm{ml}$ of whole anticoagulated blood was diluted 1:1 with sterile phosphate buffered saline (PBS) and subjected to centrifugation over a Ficoll-Hypaque density gradient (Pharmacia, Upsala Sweden) to isolate PBMC's. The isolated cells were washed 3 times in sterile PBS and resuspended in RPMI medium 1640 (Gibco BRL, Grand Island, NY) containing $10 \%$ fetal bovine serum (GIBCO) and $1 \times 10^{5} \mathrm{mg} / \mathrm{ml}$ penicillin and streptomycin (GIBCO). The final concentration of cells was determined using a hemacytometer. A final concentration ranging from $0.7-1.3 \times 10^{6} \mathrm{cells} / \mathrm{ml}$ was used in all experiments. For intracellular cytokine analysis cells were cultured in either medium containing $10 \mathrm{ng} / \mathrm{ml} \mathrm{PMA} \mathrm{and} 1 \mathrm{mg} / \mathrm{ml}$ ionomycin for 5 hours or medium containing $10 \mathrm{ug} / \mathrm{ml}$ with $5 \mu \mathrm{g} / \mathrm{ml}$ lipopolysaccharide E.coli strain 011:B4 (LPS; Sigma). In all cases $3 \mathrm{mM}$ monensin (Sigma) was added to the PBMC cultures to shut down extracellular transport of cytokines and allow intracellular accumulation. For assessment of the cell surface activation markers CD69 and CD25 in response to stimulus isolated PBMC's were cultured in medium containing $10 \mu \mathrm{g} / \mathrm{ml}$ PHA for 24 hours.

Surface marker staining. For WBC surface marker analysis $100 \mu$ l of peripheral whole blood was stained along with the manufactures suggested amount of FLl:fluorescein isothiocyanate (FITC), FL2:phycoerythrin (PE) or FL3:Peridinin Chlorophyll Protein (PercP) labeled monoclonal antibodies in $100 \mu$ l of phosphate buffered saline (PBS). The mixtures were incubated on ice for 15 minutes, $3 \mathrm{ml}$ of $.85 \%$ ammonium chloride was added and the mixture was incubated in the dark for 5 minutes to lyse the RBC's, washed 3 times with $3 \mathrm{ml}$ of PBS and resuspended in $0.5 \mathrm{ml}$ PBS with $1 \%$ paraformaldehyde.

For the assessment of CD69 and CD25 the cells (activated in culture) were washed and resuspended in 500 $\mu$ l PBS to which the manufacturers suggested amount of labeled monoclonal antibodies to CD69 and CD25 were added. Following incubation for 10 minutes the cells were washed and resuspended in $500 \mu$ l of PBS containing $1.0 \%$ paraformaldehyde prior to analysis. 
Intracellular cytokine staining. An antibody configuration was selected which assessed $T$ cell production of IFN $\gamma$ and IL-2 in T cells (including production from CD4+ and CD8+ T cell subsets) in response to PBMC stimulation with PMA and ionomycin. Surface markers were stained first by resuspending the cells in $200 \mathrm{ml}$ of PBS to which $1.0 \mathrm{mg}$ of PerCP labeled mouse monoclonal antibody to CD3, CD4 or CD8 was added, samples were incubated at R.T. for 10 minutes and then washed with PBS. The PBMC's were then fixed in $200 \mathrm{ml}$ of $4.0 \%$ paraformaldehyde in PBS for 10 minutes, then washed again in PBS. To detect intracellular production of IFN $\gamma$ or IL-2 in conjunction with a surface marker, fixed PBMC's were resuspended in $200 \mathrm{ml}$ of permeabilization buffer (PB), consisting of $5.0 \%$ non-fat dry milk and $0.5 \%$ saponin in PBS to which $0.5 \mathrm{mg}$ of labeled mouse antibody to either IFN $\gamma$ or IL-2 was added. Cells were incubated at room temperature for 25 minutes. The cells were then washed in PBS containing saponin (PBSS) and resuspended in $1.0 \%$ paraformaldehyde for analysis.

To detect intracellular production of IL- $1 \alpha$ in conjunction with surface CD14 in monocytes, the activated PBMC's were resuspended in $200 \mathrm{ml}$ of PBS to which $1.0 \mathrm{mg}$ of FITC labeled mouse monoclonal antibody to CD 14 was added and incubated at R.T. for 10 minutes. The PBMC's were then washed in PBS and fixed in $200 \mathrm{ml}$ of $4.0 \%$ paraformaldehyde in PBS for 10 minutes, then washed again in PBS. To detect intracellular IL- $1 \alpha$, the fixed PBMC's were resuspended in $200 \mathrm{ml}$ of PB with $0.5 \mathrm{mg}$ of a PE labeled antibody to IL- $1 \alpha$ (Pharmingen), incubated for 25 minutes at R.T. and washed with PBSS. The cells were then resuspended in $1.0 \%$ paraformaldehyde for analysis.

Flow cytometry analysis. For analysis, a Coulter XL flow cytometer was configured with appropriate electronic compensation for spectral overlap of the light emitted by the different fluorochromes. The lymphocyte cluster was identified by plotting forward versus side $\left(90^{\circ}\right)$ light scatter. The lymphoid cells were then 'gated' for immunofluorescence analysis, including only the lymphoid cells in the data analysis. Thresholds for positive were set using isotype matched control antibodies. Sample analysis was performed using System 2 software on a Coulter-XL flow cytometer

Serum and urine stress hormone measurement. Analysis of serum and 24-hour urine were performed using commercially available kits according to the manufacturer's instructions. Serum/urine cortisol were performed by RIA (DiaSorin, Stillwater, MN); urine aldosterone was performed by RIA (Diagnostic Product Corporation, Los Angeles, CA); urine ADH was perfomed by RIA (Diagnostic Systems Laboratories, Inc., Webster, TX); urine epinephrine/norepinephrine were performed by RIA (ALPCO, Windham, NH).

Statistical analysis. To test the significance of differences observed as a result of exposure to microgravity either a one way repeated measures analysis of variance (three groups) or a paired $\mathrm{T}$ test (two groups) was performed. The results obtained from preflight (L-10) testing were compared to the results obtained from landing day $(\mathrm{R}+0)$ testing. Statistical significance was defined by $\mathrm{P}<0.05$ and differences which achieved significance are indicated as such in the figures. 


\section{RESULTS}

White Blood Cell Differential. The data were consistent with significant alterations in the status of the peripheral immune system upon landing. An analysis of relative percentages of granulocytes, lymphocytes and monocytes from all four shuttle flights indicated a statistically significant increase in the percentage of peripheral blood granulocytes, which was accompanied by a corresponding significant decrease in the relative percentage of peripheral blood lymphocytes (data not shown). Considering that the percentages of WBC subsets are relative with respect to each other, an analysis of absolute peripheral blood cell counts was performed. Absolute levels of peripheral granulocytes were significantly elevated following spaceflight, but the levels of circulating lymphocytes and monocytes were unchanged (Fig. 1). These data indicate that the differences in circulating granulocytes had artificially skewed the lymphocyte percentage data. After three days of exposure to unit gravity, the percentages in most of the subjects had returned to near baseline. No significant alterations regarding levels of circulating monocytes were seen following spaceflight (Fig. 1).

Surface Marker Subset Analysis. Lymphocyte subset analysis demonstrated a significantly decreased percentage of $\mathrm{T}$ cells $(\mathrm{CD} 3+)$ in 10 of 13 subjects $(\mathrm{P}<0.05$, Fig. 2a) and a slightly increased percentage of $B$ cells (CD19+) in 9 of 13 subjects (Fig. 2b). No real trend was seen in the percentage of NK cells (CD16/56+; Fig. 2c). Nearly all (23 of 27) astronauts had an increased CD4:CD8 ratio, which was dramatic in some individuals $(P<0.05$, Fig. 2d). For the majority of the subjects with an increased CD4:CD8 ratio at $R+1$, the ratios returned to near baseline (L-10) values within three days after landing $(R+3)$. Assessment of memory $(C D 45 R A+)$ vs. naïve $(\mathrm{CD} 45 \mathrm{RO}+) \mathrm{CD} 4+\mathrm{T}$ cell subsets was more ambiguous, with subjects tending to group as a flight crew. All subjects from one mission ( 7 of 7 ) demonstrated an increased CD45RA:CD45RO ratio, while all subjects from another mission (5 of 5 ) demonstrated a decreased ratio (Fig. 2e).

Subjects from a third mission exhibiteded no significant trend. The reason for this grouping is unclear. While no significant trend was seen in the monocyte population as defined by scatter, a significant decrease in the percentage of CD14+CD16+ monocyte subset was seen in all subjects tested $(P<0.05$, Fig. $2 \mathrm{f})$. All of the cellular changes described above which were assessed at $R+0$ trended to pre-flight levels by $\mathrm{R}+3$.

Expression of Cellular Activation Markers. Isolated PBMC's from astronaut whole blood samples were cultured in the presence of $10 \mu \mathrm{g} / \mathrm{ml}$ PHA for 24 hours to assess the capacity of the cells to respond to a mitogenic signal. Under normal conditions, the expression of CD69 occurs early during lymphocyte activation, and the expression of CD25 occurs somewhat later during the activation process. No significant differences were seen in the expression of the cellular activation markers CD69 and CD25 following activation in culture for 24 hours at either $\mathrm{R}+0$ or $\mathrm{R}+3$ as compared to L-10 preflight samples (data not shown).

Intracellular Cytokine Detection by Flow Cytometry. Significant alterations in cytokine production by specific lymphocyte subsets in response to mitogenic activation were detected by flow cytometry. Representative flow cytometry scatter plots demonstrating decreased production of IFN $\gamma$ and IL-2 after spaceflight $(\mathrm{R}+0)$, as compared to preflight (L-10) and a three day recovery after flight $(R+3)$ for one astronaut are presented in figure 3 . The mean percentages of cytokine producing cells for all timepoints were determined and are presented in figure 4 . In the grouped subjects, the data indicate a suppression in the ability of stimulated T cells $(\mathrm{CD} 3+)$ to 
produce IL-2 after spaceflight. T cell production of IFN $\gamma$ was not affected. When cytokine production by the individual $\mathrm{T}$ cell subsets (CD4+ and $\mathrm{CD} 8+$ ) was examined,

the suppression of IL-2 production was evident in both the CD4 and the CD8 T cell subsets

(figure 4). In contrast, IFN $\gamma$ production was reduced in the CD4+ $\mathrm{T}$ cell subset while CD8+ $\mathrm{T}$ cell production of IFN $\gamma$ appeared to be unchanged.

To examine the subjects individually, the cytokine production data for the astronauts at L-10 was normalized to $100 \%$ (Fig. 5). Thus, the relative increases or decreases at $R+0$ may be visualized for all subjects while maintaining the ability to look at individual data points. $T$ cell (CD3+) production of IL-2 was decreased after at $R+0$ in all subjects assayed ( 7 of 7 ; Fig. 5a). In these same subjects, however, no trend emerged regarding the $\mathrm{T}$ cell expression of IFN $\gamma$ (Fig. 5d). Specific T cell subset (CD4 vs. CD8) cytokine production was assessed for a larger population of astronauts. It was found that the ability to produce IL-2 in response to mitogenic stimulus was depressed for both the CD4+ subset (11 of 12 subjects; Fig 5b) and for the CD8+ subset ( 18 of 20 subjects; Fig $\mathbf{5 c}$ ).

Generalized $\mathrm{T}$ cell $(\mathrm{CD} 3+)$ production of IFN $\gamma$ was altered, although this assay was performed on only one mission with a sample size of seven. The production of IFN $\gamma$ by specific $T$ cell subsets was investigated on a larger sample size spanning three missions. When $T$ cell production of IFN $\gamma$ was assessed in the context of CD4+ and CD8+ subsets, the CD4+ T cell subset appeared to be more sensitive to the effects of spaceflight. The majority of astronauts tested demonstrated a decrease in production ( 10 of $12 ; \mathrm{p}<0.05$; Fig. 5e) of IFN $\gamma$. The production of IFN $\gamma$ by CD8+ T cells was effected by spaceflight as no clear trend emerged (Fig. $5 \mathrm{f}$ ).

The ability of monocytes to produce IL- $1 \alpha$ in response to mitogenic stimulus was also assessed. No dramatic alterations and no clear trends emerged for the majority of the subjects (14 of 20; data not shown), however for a small number of subjects (6 of 20) a dramatic increase in $\mathrm{IL}-1 \alpha$ production was seen at $\mathrm{R}+0$. For these subjects these responses trended towards $\mathrm{L}-10$ levels by $R+3$. This population represented the entire crew for one space shuttle flight out of the three flights for which this assay was performed. A comparison of the mean percentage of IL-1 $\alpha$ producing monocytes for $\mathrm{L}-10$ and $\mathrm{R}+0$ indicated the increase in production was statistically significant $(\mathrm{p}<0.05)$.

Serum and urine chemistries. Serum and urine stress hormone analysis was performed on subjects both pre-flight and immediately post-flight to investigate the physiologic stress resulting from spaceflight/landing. Serum cortisol and ACTH, as well as urine cortisol, aldosterone, ADH, epinipherine and norepinepherine were assessed. All mean measurements were elevated postflight, as compared to preflight values (Fig. 6). The elevations for urine cortisol, aldosterone and norepinepherine achieved statistical significance $(P<0.05$, Fig .6$)$. These results indicate that the astronaut subjects were subjected to significant physical stresses during, or immediately following spaceflight. 


\section{DISCUSSION}

The dysregulation of the immune system is a well-documented result of prolonged exposure to microgravity. Several studies have identified altered cytokine production profiles in subjects exposed to microgravity for prolonged periods $(9,10,11)$. In addition, ground based studies have also indicated that simulated microgravity also results in a depressed immune response and reduced capacity to produce specific cytokines $(2,3,4,5,6,7)$.

Here we confirm the findings of others regarding alterations in the peripheral blood immune cell subsets. In particular, we demonstrate an increase in the percentage of peripheral blood granulocytes and a decrease in percentages of lymphocytes immediately following spaceflight. This is consistent with the results seen by Stowe, et al who recently reported on neutrophil percentages and function following spaceflight (25). Since the percentages of WBC subsets are relative with respect to each other, an analysis of absolute peripheral blood cell counts was performed. Absolute levels of peripheral granulocytes were significantly elevated following spaceflight and the absolute levels of lymphocytes and monocytes were unchanged (Fig. 1). These data indicate that the differences in circulating granulocytes had artificially skewed the lymphocyte percentage data. Regarding specific lymphocyte subsets, we report here that the $T$ cell CD4:CD8 subset ratio increased following spaceflight. It is likely that this result would be attributed to decreased levels of CD8 $+\mathrm{T}$ cells, as opposed to increased levels of CD4+ T cells, given the overall decrease in the levels of total $\mathrm{T}$ cells. These results will require confirmation in the future on a larger sample size and perhaps using recently developed methods to directly quantify absolute counts during flow cytometry analysis. No significant trends emerged regarding levels of B cells and NK cells following spaceflight in our study. In addition, ratios of memory (CD45RO+) to naïve (CD45RA+) T cells did not demonstrate significant trends in our study. This was not unexpected, as the duration of these flights was probably not long enough to result in a broad expansion of memory cells, and since no significant lengthy illnesses were reported during these four shuttle flights. No alterations in monocyte levels were detected when analyzing by scatter properties alone. The recently described subset of CD 14+/CD16+ peripheral blood monocytes was also assessed in this study. This unique subset, functionally appearing like tissue macrophages, has recently been identified and associated with specific disease processes $(26,27)$. This subset comprises approximately $10 \%$ of the total peripheral blood monocyte population. Our preflight CD14+CD16+ monocyte values correlated well with that value, and a significant decrease was observed postflight. Values rebounded to levels at or above the preflight value by $\mathrm{R}+3$. Although the clinical significance of this finding is unknown, this subset has been reported to be proinflammatory in nature. Levels were reported to be decrease in subjects after extreme exercise (26). This decrease may be an acute response to the physical stresses of landing. Inflight measures will be required to further investigate the role of these cells in spaceflight immune responses.

In this study we have identified specific subpopulations of immune cells which demonstrate alterations in their ability to produce cytokines. This was accomplished by utilizing flow cytometry, with which cytokines and surface markers can be stained simultaneously, resulting in positive identification of cytokine producing cells. This technology was used to positively identify the particular subsets of T cells susceptible to spaceflight-induced cytokine (IFNy and IL-2) dysregulation. We report here that the ability of $T$ cells to produce IL-2 was depressed in the total $\mathrm{T}$ cell population, as well as in both the CD4+ subset and the CD8+ subset. This alteration was statistically significant for all three populations, and results trended towards preflight values in the $R+3$ specimens. This indicates that a recovery in IL-2 responsiveness was occurring several 
days after landing. An interesting differentiation in the effects of spaceflight was observed for the $\mathrm{T}$ cell subsets regarding the production of IFN $\gamma$. While total $\mathrm{T}$ cell production of IFN $\gamma$ was not effected in a significant fashion (although tested in a notably small sample size), the CD4+ subset did demonstrate a significant reduction in IFN $\gamma$ production. This effect was not observed in the $\mathrm{CD} 8+$ subset. Although the individualized data plot for CD4+/IFN $\gamma$ production reveals 5 of 20 astronauts with increased production after spaceflight, statistical analysis of the entire population demonstrated a significant reduction overall (with 15 of 20 astronauts demonstrating a reduction for this subset). In the context of the increased CD4:CD8 ratio demonstrated earlier, it is possible that the bias of the peripheral blood CD4+ $\mathrm{T}$ cell population is being shifted away from IFNy production. Future studies investigating this phenomenon should include a comprehensive study the effect of microgravity on the Th1/Th2 cytokine balance. Further testing with increased sample size is clearly indicated to confirm these results. This data suggest that the effects of spaceflight on cytokine production may differ for both the individual cytokines and also for different cell subsets responsible for cytokine production.

It should be noted that assessments of biological activity, such as the ones conducted in this paper, on astronauts returning from spaceflight can be skewed by the psychological and physiological stresses of landing and readaptation to unit gravity. The serum and urine stress hormone results reported here indicate that subjects returning from spaceflight have been exposed to significant physical stress. The only way to overcome this potential shortcoming is to perform testing during spaceflight. Unfortunately, the technical limitation of experimentation in spaceflight has precluded performing many sophisticated laboratory analyses in the space environment. The development of newer innovative biological spaceflight-compatible hardware for performing routine immunologic monitoring may overcome some of these problems $(28,29)$.

As increased numbers of humans will be subjected to spaceflight of increasing duration, the dysregulation of the human immune system in microgravity is becoming a great concern to the spaceflight medical community. This is especially true as NASA and other space agencies prepare for lengthy missions to Mars. Thorough investigation of the effects of spaceflight on the human immune system may result in the development of effective countermeasures either via pharmacology or through manipulation of the spaceflight environment (i.e. artificial gravity, or other mechanisms). It is expected that the International Space Station will serve as an excellent platform to further extend research into the immunology effects on human physiology, as well as other related biological sciences. This data would then enhance our ability to develop required countermeasures and ensure the health and safety of future long-duration spaceflight crew members.

\section{ACKNOWLEDGMENTS}




\section{REFERENCES}

I. Sonnenfeld, G. 1998. Immune responses in space flight. Int J Sports Med. 19 Suppl 3:S 195-202; discussion S202-4.

2. Berry, W. D., J. D. Murphy, B. A. Smith, G. R. Taylor, and G. Sonnenfeld. 1991. Effect of microgravity modeling on interferon and interleukin responses in the rat. J Interferon Res. 11:243-9.

3. Walther, I., P. Pippia, M. A. Meloni, F. Turrini, F. Mannu, and A. Cogoli. 1998. Simulated microgravity inhibits the genetic expression of interleukin-2 and its receptor in mitogen-activated T lymphocytes. FEBS Lett. 436: $115-8$.

4. Uchakin PH, Cubbage ML, Sams CF, Morukov BV, Larina IV and Bobrovnik, EB. Effects of the 120 days of head-down bed rest on cytokine secretion and its in-vitro modulation by glucocorticoids. J Gravitat Physiol $5(1): 171-172$.

5. Cooper, D., and N. R. Pellis. 1998. Suppressed PHA activation of $T$ lymphocytes in simulated microgravity is restored by direct activation of protein kinase C. J Leukoc Biol. 63:550-62.

6. Cogoli A, Valluchi-Morf M, Mueller M, Briegleb W. Effect of hypogravity on human lymphocyte activation. Aviat Space Environ Med 5I(1):29-34.

7. DeGroot, RP, Rijken PJ, DenHertog J, Boonstra J, Verkleij A, DeLaat SW and Kruijer W. Nuclear responses to protein kinase $C$ signal transduction are sensitive to gravity changes. Exper Cell Res 197:87-90.

8. Taylor, G. R. 1993. Immune changes during short-duration missions. J Leukoc Biol. 54:202-8.

9. Grove, D. S., S. A. Pishak, and A. M. Mastro. 1995. The effect of a 10-day space flight on the function, phenotype, and adhesion molecule expression of splenocytes and lymph node lymphocytes. Exp Cell Res. 219:1029.

10. Nash, P. V., I. V. Konstantinova, B. B. Fuchs, A. L. Rakhmilevich, A. T. Lesnyak, and A. M. Mastro. 1992. Effect of spaceflight on lymphocyte proliferation and interleukin-2 production. J Appl Physiol. 73: 186S$190 \mathrm{~S}$.

11. Sonnenfeld, G., C. L. Gould, J. Williams, and A. D. Mandel. 1988. Inhibited interferon production after space flight. Acta Microbiol Hung. 35:411-6.

12. Meehan, R. T., L. S. Neale, E. T. Kraus, C. A. Stuart, M. L. Smith, N. M. Cintron, and C. F. Sams. 1992. Alteration in human mononuclear leucocytes following space flight. Immunology. 76:491-7.

13. Taylor, G. R., L. S. Neale, and J. R. Dardano. 1986. Immunological analyses of U.S. Space Shuttle crewmembers. Aviat Space Environ Med. 57:213-7.

14. Konstantinova, I. V., M. P. Rykova, A. T. Lesnyak, and E. A. Antropova. 1993. Immune changes during long-duration missions. J Leukoc Biol. 54:189-20l.

15. Taylor, G. R., and R. P. Janney. 1992. In vivo testing confirms a blunting of the human cell-mediated immune mechanism during space flight. J Leukoc Biol. 51:129-32.

16. Gumnder FK, Konstantinova I, Cogoli A, Lesnyak A, Bogomolov W, Grachovv A W. 1994. Cellular immunity in cosmonauts during long duration spaceflight on board the orbital MIR station. Aviat Space Environ Med. 65(5):419-423. 
17. Sander, B., J. Andersson, and U. Andersson. 1991. Assessment of cytokines by immunofluorescence and the paraformaldehyde- saponin procedure. Immunol Rev. 119:65-93.

18. Jung, T., U. Schauer, C. Heusser, C. Neumann, and C. Rieger. 1993. Detection of intracellular cytokines by flow cytometry. J Immunol Methods. 159:197-207.

19. Prussin, C., and D. D. Metcalfe. 1995. Detection of intracytoplasmic cytokine using flow cytometry and directly conjugated anti-cytokine antibodies. J Immunol Methods. 188:117-28.

20. Elson, L. H., T. B. Nutman, D. D. Metcalfe, and C. Prussin. 1995. Flow cytometric analysis for cytokine production identifies $\mathrm{T}$ helper 1 , $\mathrm{T}$ helper 2 , and $\mathrm{T}$ helper 0 cells within the human $\mathrm{CD} 4+\mathrm{CD} 27-$ lymphocyte subpopulation. J Immunol. 154:4294-301.

21. Picker, L. J., M. K. Singh, Z. Zdraveski, J. R. Treer, S. L. Waldrop, P. R. Bergstresser, and V. C. Maino. 1995. Direct demonstration of cytokine synthesis heterogeneity among human memory/effector $T$ cells by flow cytometry. Blood. 86: 1408-19.

22. Maino, V. C., and L. J. Picker. 1998. Identification of functional subsets by flow cytometry: intracellular detection of cytokine expression. Cytometry. 34:207-15.

23. Jung, T., G. Lack, U. Schauer, W. Uberuck, H. Renz, E. W. Gelfand, and C. H. Rieger. 1995. Decreased frequency of interferon-gamma- and interleukin-2-producing cells in patients with atopic diseases measured at the single cell level. J Allergy Clin Immunol. 96:515-27.

24. Crucian, B., P. Dunne, H. Friedman, R. Ragsdale, S. Pross, and R. Widen. 1996. Detection of altered T helper 1 and $T$ helper 2 cytokine production by peripheral blood mononuclear cells in patients with multiple sclerosis utilizing intracellular cytokine detection by flow cytometry and surface marker analysis. Clin Diagn Lab Immunol. 3:411-6.

25. Stowe RP, Sams CF, Mehta SK, Kaur I, Jones ML, Feeback DL, Pierson DL. 1999. Leukocyte subsets and neutrophil function after short-term spaceflight. J. Leukoc. Biol. 65(2):179-186.

26. Ziegler-Heitbrock H.W.L. 1996. Heterogeneity of human blood monocytes: the CD14+ CD16+ subpopulation. Immunol. Today 17(9):424-428.

27. Fingerle G., Pforte A., Passlick B., Blumenstein M., Strobel M. and Ziegler-Heitbrock H.W.L. 1993. The novel subset of CD 14+/CD 16+ blood monocytes is expanded in sepsis patients. Blood 82(10):3170-3176.

28. Sams, C, Crucian, B., Cubbage M. and E. Meinelt. (1999) Development of a whole blood staining device for use during space shuttle flights. Cytometry 37:74-80.

29. Crucian B. and C. Sams. (1999) The use of a spaceflight-compatible device to perform WBC surface marker staining and mitogenic activation for cytokine detection by flow cytometry. Joumal of Gravitational Physiology 6(1):33-34. 
Figure Legends

Figure 1. Absolute white blood cell counts 10 days prior to spaceflight ( $L-10)$, landing day $(R+0)$ and 3 days after landing $(\mathrm{R}+3)$. Levels are expressed as $10(3)$ cells/microliter. Significant differences $(\mathrm{P}<0.05)$ are indicated by $\left.{ }^{*}\right)$. Data are plotted with error presented as standard error of the mean.

Figure 2. Percentages of white blood cell subsets 10 days prior to spaceflight $(L-10)$, landing day $(R+0)$ and 3 days after landing $(R+3)$. The subsets assessed were (A) T cells, (B) B cells, (C) NK cells, (D) CD4:CD8 ratio, (E) CD45RA:CD45RO ratio and $(F)$ CD 14+/CD 16+ monocyte subset. To assess alterations induced by spaceflight, the preflight (L-10) percentages have been normalized to 100 percent, with the $R+0$ and $R+3$ percentages adjusted accordingly.

Figure 3. Representative flow cytometry histograms for one astronaut subject demonstrating percentages of CD4+ and CD8 $+T$ cells being capable of being stimulated to produce IFN $\gamma$ and $\mathbb{L}-2$. Assessments were made 10 days prior to spaceflight $(\mathrm{L}-10)$, landing day $(\mathrm{R}+0)$ and 3 days after landing $(\mathrm{R}+3)$.

Figure 4. Mean percentages for all astronaut subjects of $T$ cells (CD3+) and $T$ cell subsets (CD4+ and CD8+) capable of being stimulated to produce the cytokines (A) $\mathbb{L}-2$ and (B) IFNy. Significant differences $(\mathrm{P}<0.05)$ are indicated by $\left(^{*}\right)$. Data are plotted with error presented as standard error of the mean.

Figure 5. Individual subject percentages for all astronaut of $T$ cells (CD3+) and $T$ cell subsets (CD4+ and CD8+) capable of being stimulated to produce the cytokines (A) IL-2 and (B) IFN $\gamma$. To assess alterations induced by spaceflight, the preflight (L-10) percentages have been normalized to 100 percent, with the $R+0$ and $R+3$ percentages adjusted accordingly. Significant differences $(\mathrm{P}<0.05)$ are indicated.

Figure 6. Serum (cortisol and ACTH) and urine (cortisol, aldosterone, ADH, epinepherine, norepinepherine) stress hormone levels following spaceflight. Serum or 24-hour urine measurements were made for the indicated preflight $(\mathrm{L}-10)$ or postflight $(\mathrm{R}+0)$ timepoint. Significant differences $(\mathrm{P}<0.05)$ are indicated by $\left({ }^{*}\right)$. Data are plotted with error presented as standard error of the mean. 


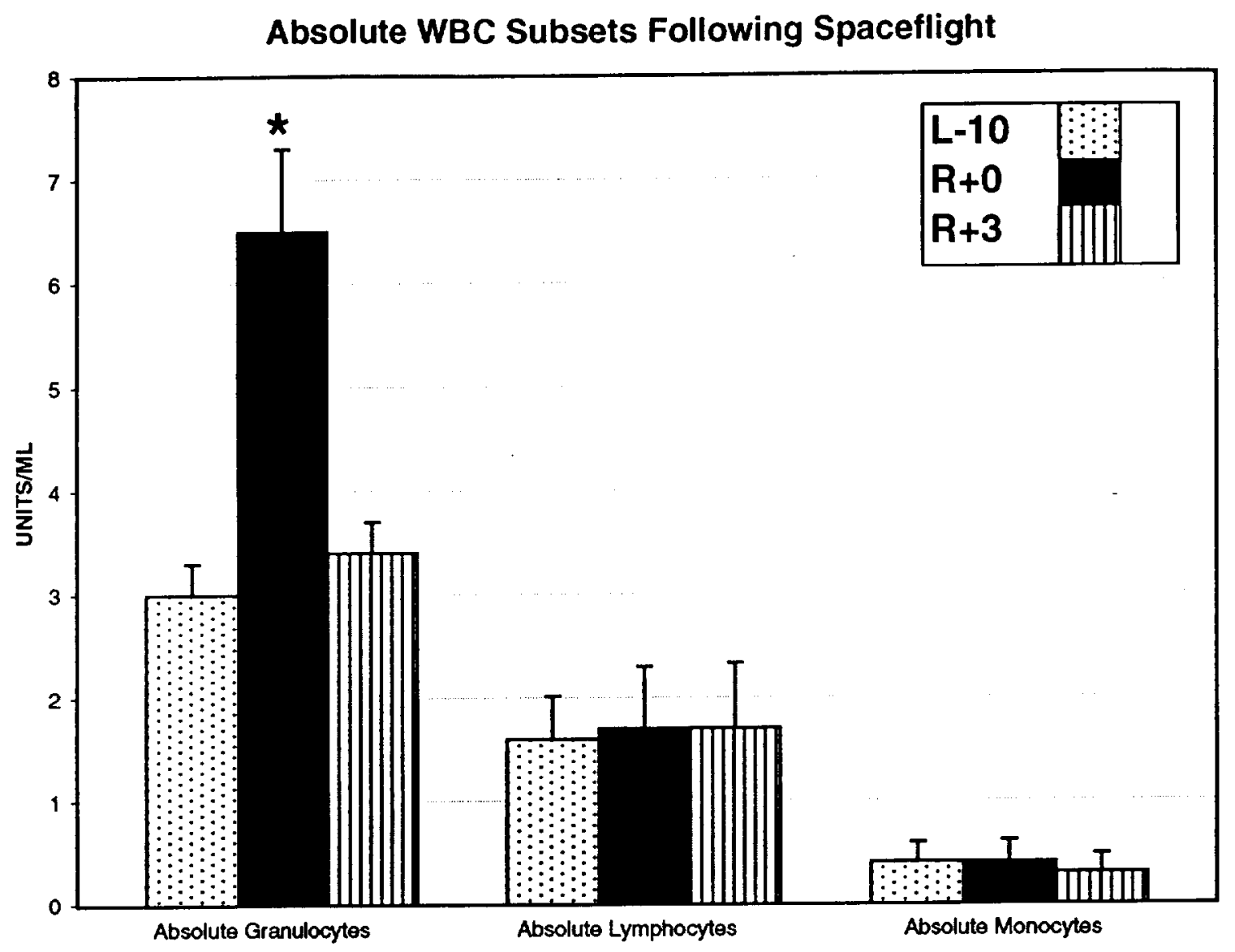

FIGURE 1 

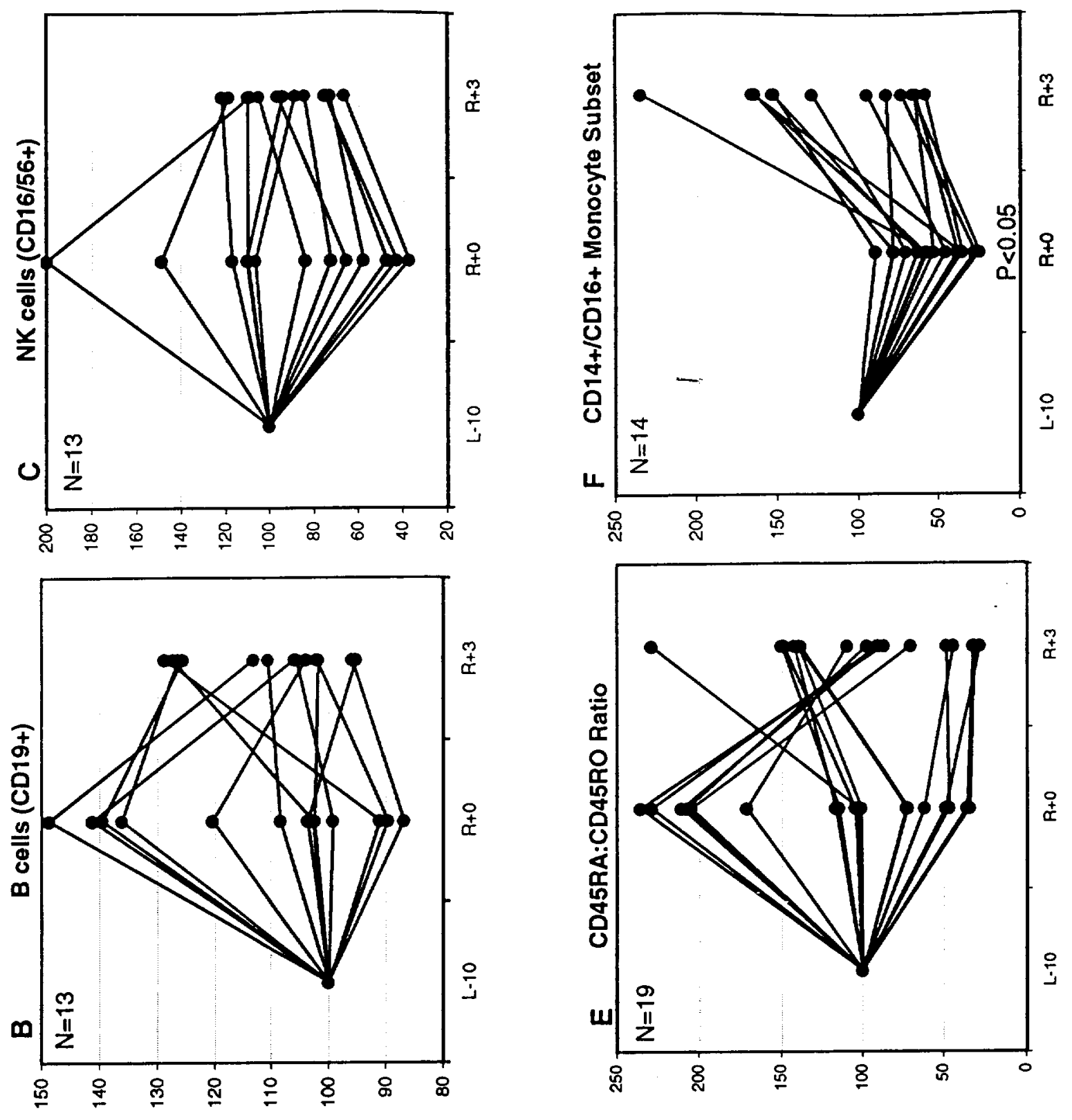

N
o
$\frac{9}{4}$
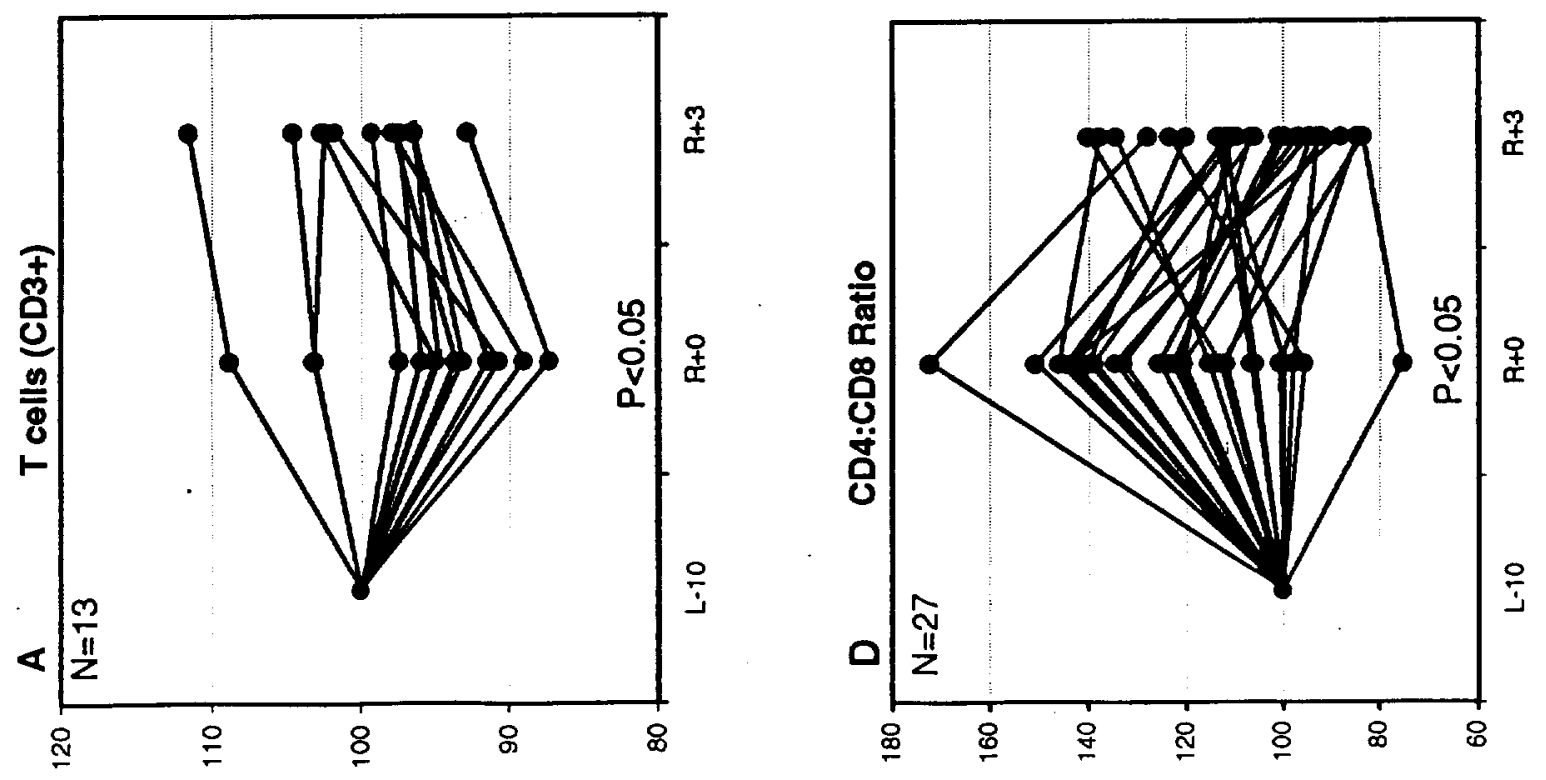

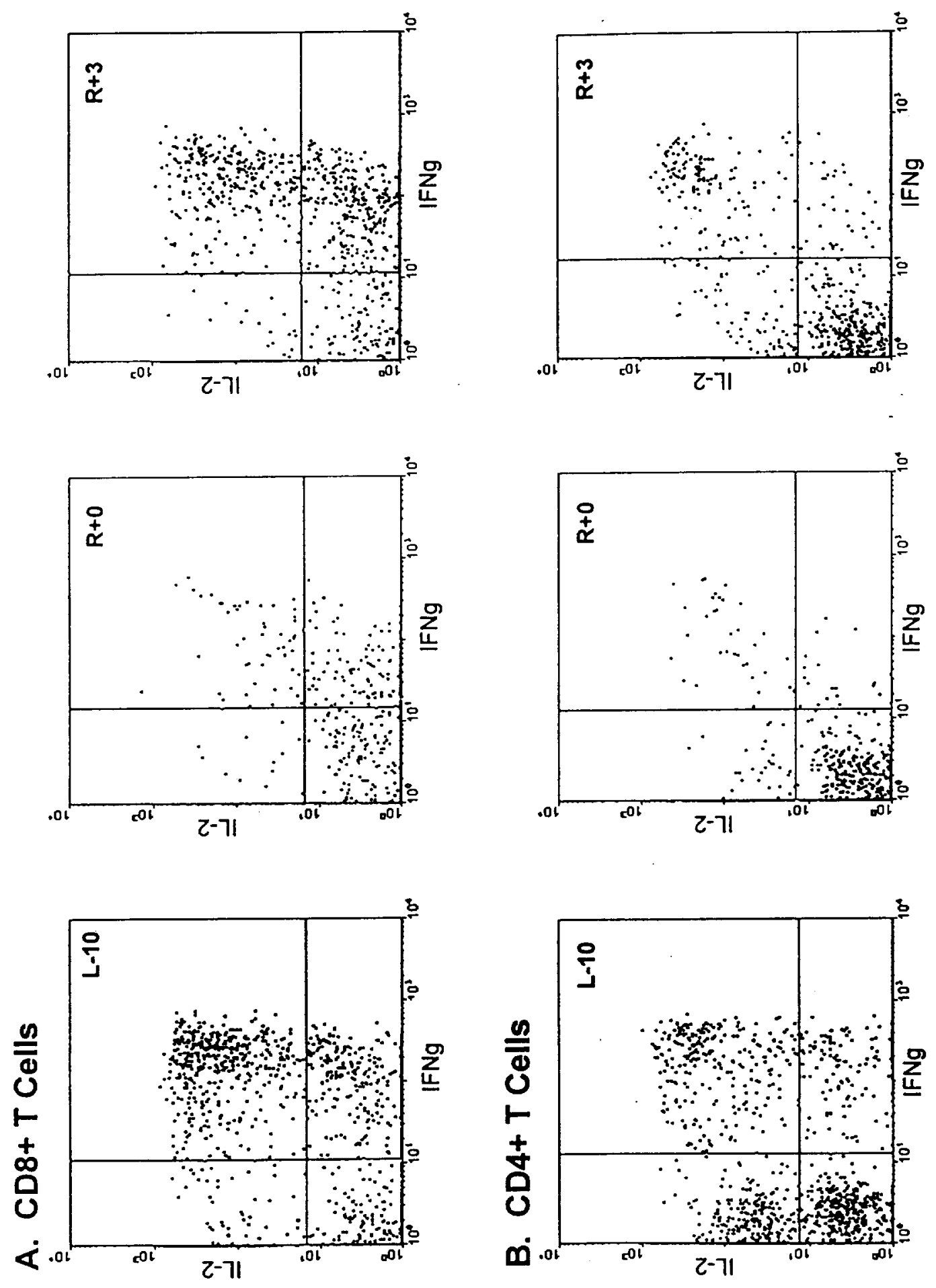

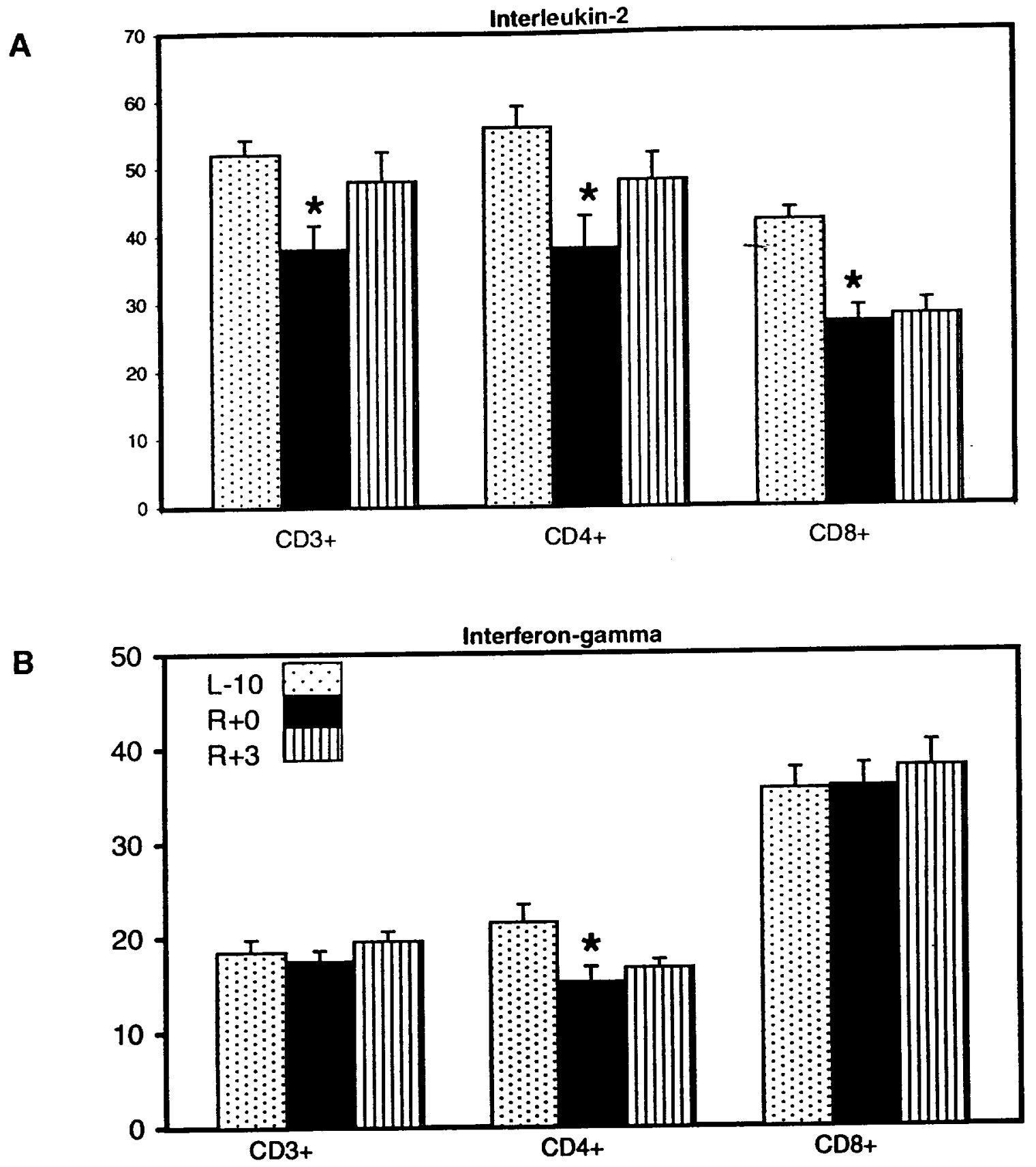

FIGURE 4 

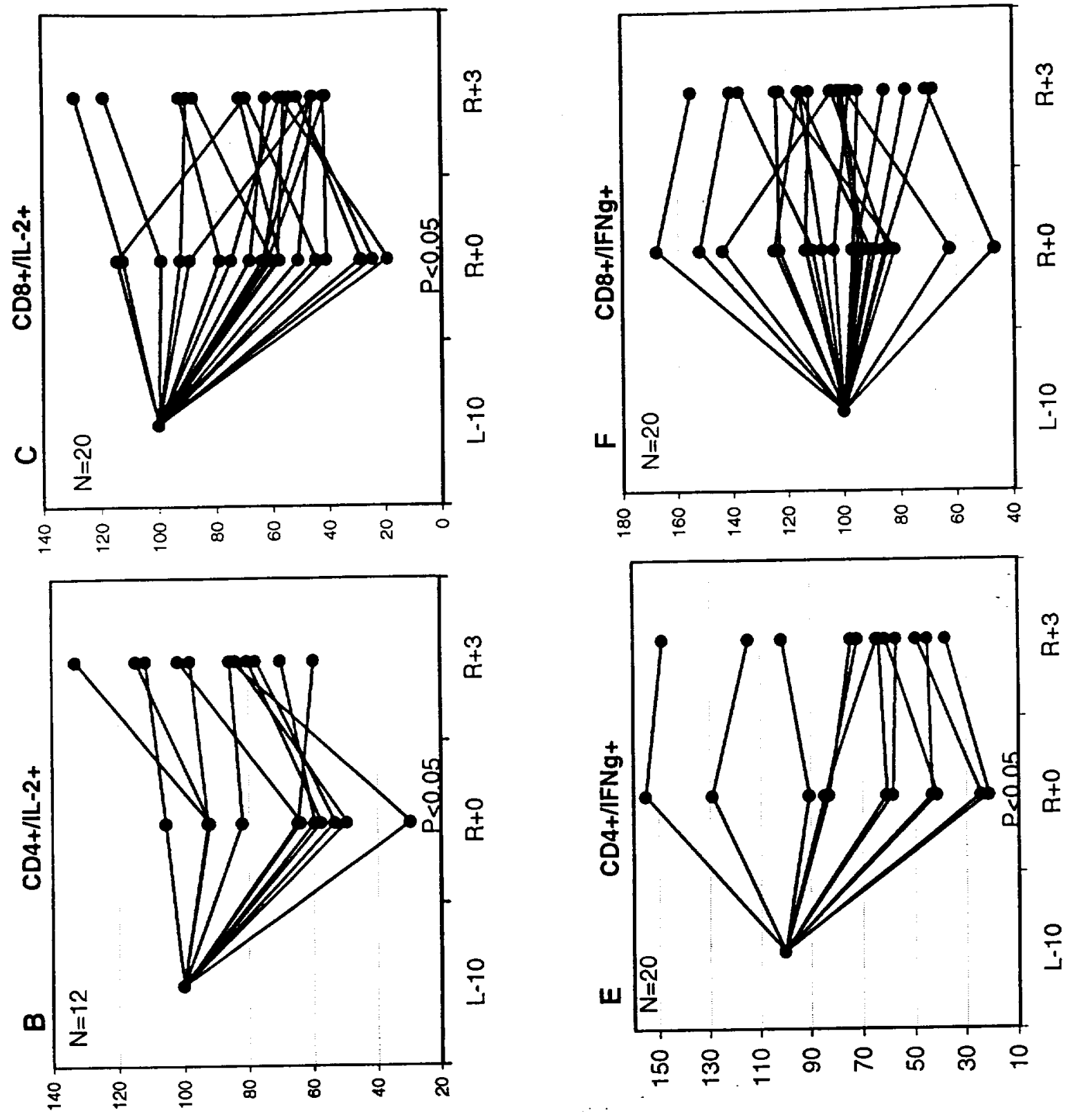

옴
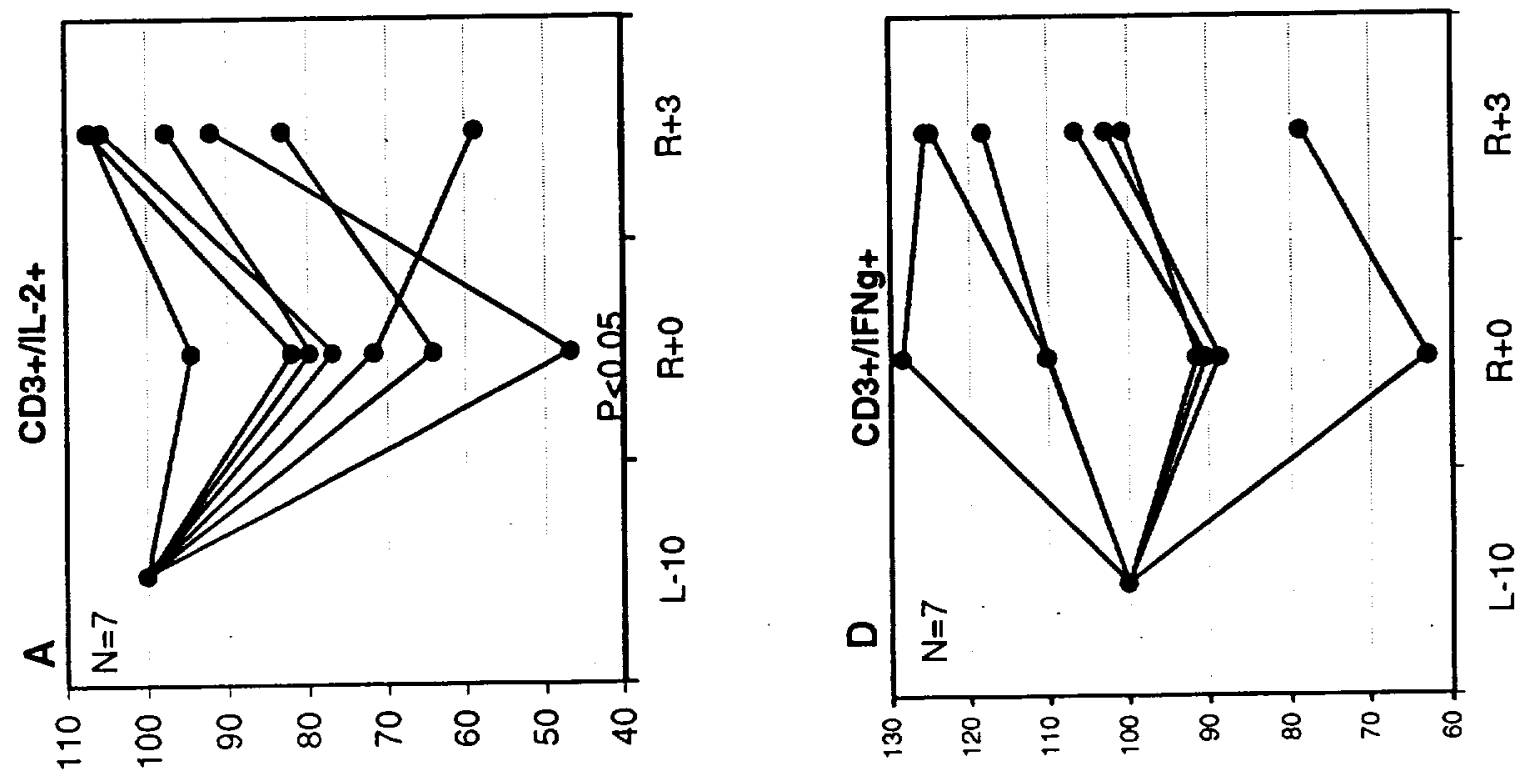


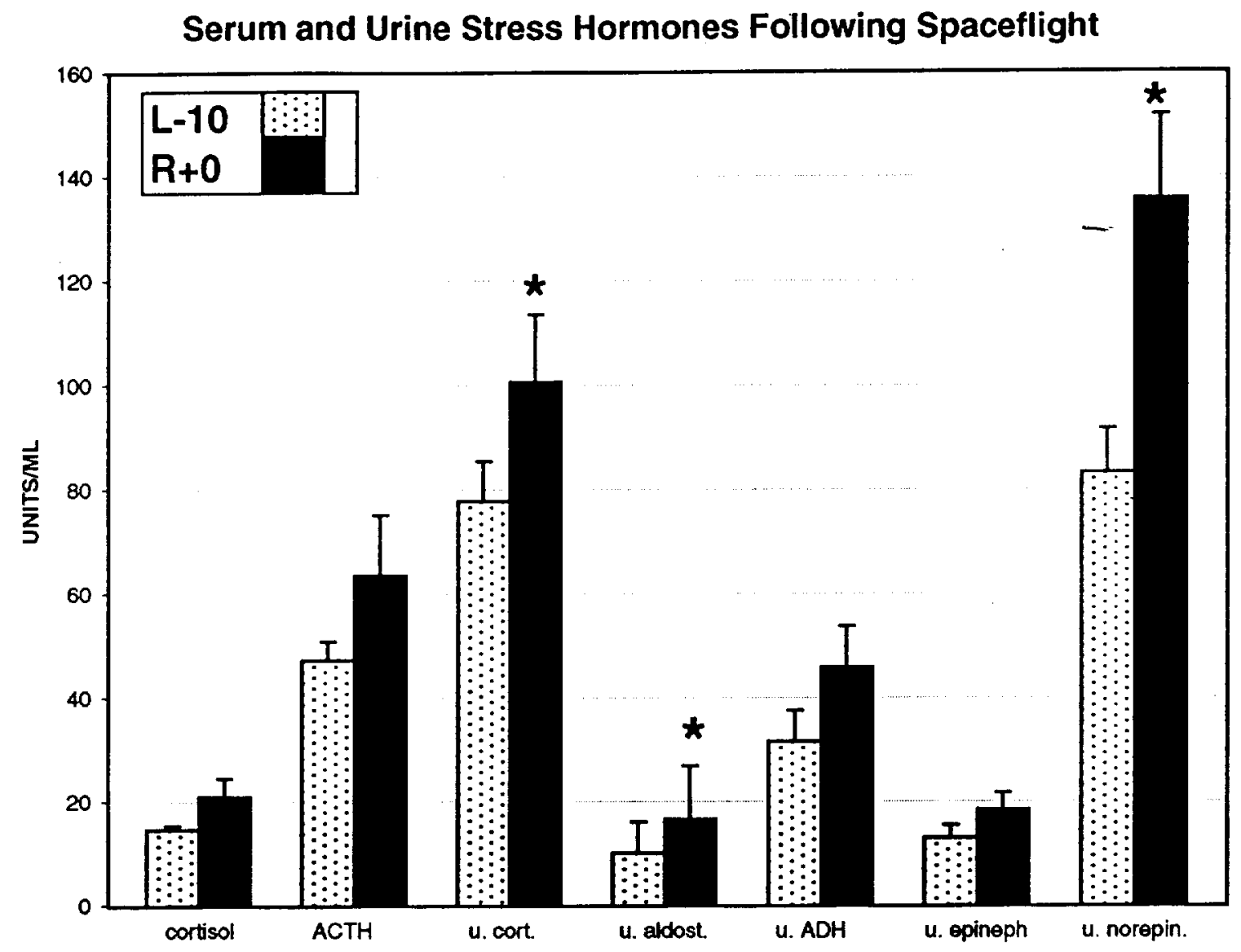

Figure 6 